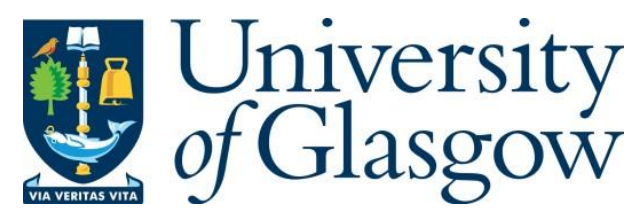

Freeman, E. and Wilson, G. (2021) Perception of Ultrasound Haptic Focal Point Motion. In: 23rd ACM International Conference on Multimodal Interaction (ICMI '21), Montréal, QC, Canada, 18-22 Oct 2021, pp. 697-701. ISBN 9781450384810 (doi:10.1145/3462244.3479950).

There may be differences between this version and the published version. You are advised to consult the publisher's version if you wish to cite from it.

(C) The Author 2021. This is the author's version of the work. It is posted here for your personal use. Not for redistribution. The definitive Version of Record was published in Proceedings of the 23rd ACM International Conference on Multimodal Interaction (ICMI '21), Montréal, QC, Canada, 18-22 Oct 2021, pp. 697-701. ISBN 9781450384810 .

http://eprints.gla.ac.uk/249297/

Deposited on: 10 August 2021

Enlighten - Research publications by members of the University of Glasgow http://eprints.gla.ac.uk 


\section{Perception of Ultrasound Haptic Focal Point Motion}

\author{
Euan Freeman \\ University of Glasgow \\ Glasgow, Scotland \\ euan.freeman@glasgow.ac.uk
}

\begin{abstract}
Ultrasound haptic patterns can be rendered by continuously moving an ultrasonic focal point. It is not known how this focal point motion affects haptic perception. We present two psychophysical experiments investigating the perception of an ultrasound haptic focal point moving along a circular path. Our first experiment finds that a sensation of motion is perceived at speeds up to $17 \mathrm{rev} \mathrm{s}^{-1}$, similar to the 'flutter' sensation. Our second experiment found a linear relationship between movement speed and perceived intensity up to this speed. Our findings give new insight into ultrasound haptic perception and give designers empirical evidence to inform contactless haptic feedback design.
\end{abstract}

\section{CCS CONCEPTS}

- Human-centered computing $\rightarrow$ Haptic devices.

\section{KEYWORDS}

haptic perception, spatiotemporal modulation, ultrasound haptics

\section{ACM Reference Format:}

Euan Freeman and Graham Wilson. 2021. Perception of Ultrasound Haptic Focal Point Motion. In Proceedings of the 2021 International Conference on Multimodal Interaction (ICMI '21), October 18-22, 2021, Montréal, QC, Canada. ACM, New York, NY, USA, 5 pages. https://doi.org/10.1145/3462244.3479950

\section{INTRODUCTION}

Ultrasound haptic devices create tactile feedback in mid-air, without the need for users to wear or touch a physical device. Early versions of this technology could produce a single ultrasonic focal point in a fixed position above the device [22]; state of the art devices are now capable of positioning and moving points in 3D [19], can create multiple focal points [5, 29], provide auditory effects $[8,18]$, and can create complex patterns with high spatial and temporal resolution [9, 12, 17, 32, 36, 37].

Ultrasonic focal points impart pressure against the skin, although this is insufficient for clear perception. In early work, amplitude was modulated to enhance perception by stimulating rapidly-adapting touch receptors [22], typically at $200 \pm 50 \mathrm{~Hz}$ (although can be perceived at least as low as $16 \mathrm{~Hz}$ [31]). Recent research has modulated the position of focal points instead, a method called lateral modulation [35, 37] and spatiotemporal modulation [12]. For example, this can be used to create a haptic circle pattern by moving a focal point along a circular trajectory (Figure 1). This allows amplitude to be

ICMI '21, October 18-22, 2021, Montréal, QC, Canada

(c) 2021 Copyright held by the owner/author(s). Publication rights licensed to ACM. This is the author's version of the work. It is posted here for your personal use. Not for redistribution. The definitive Version of Record was published in Proceedings of the 2021 International Conference on Multimodal Interaction (ICMI '21), October 18-22, 2021, Montréal, QC, Canada, https://doi.org/10.1145/3462244.3479950.

\author{
Graham Wilson \\ University of Strathclyde \\ Glasgow, Scotland \\ graham.a.wilson@strath.ac.uk
}

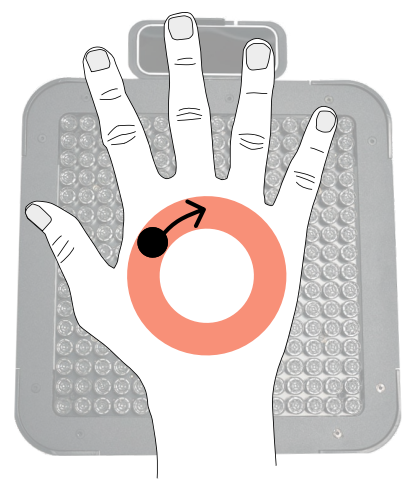

Figure 1: Mid-air haptic patterns can be created by rapidly moving ultrasonic focal points, e.g., along a circular path.

maintained without modulation, leading to more intense feedback than amplitude modulated patterns [12,37].

A novel characteristic of spatiotemporal modulation is that it stimulates touch receptors through continuous rapid movement. Focal points are typically moved at high speeds, with the highest intensity for circular patterns occurring around $7 \mathrm{~m} \mathrm{~s}^{-1}$ [12]. At such speeds, the motion itself is imperceptible; instead, users feel a contiguous pattern against their hand. It is not known how well focal point movement itself is perceived at lower speeds.

In this paper, we describe two experiments investigating the perceived sensation of motion from ultrasound haptic patterns. We focus on circular trajectories, because they are continuous and have no overlapping points or gaps. They are also widely used in ultrasound haptic interfaces [32]. Our first experiment investigates the effect of focal point movement on perception of 'motion'. Our second experiment investigates the relation between render frequency and perceived magnitude, below the threshold for perceived motion. Our results from these robust psychophysical studies give data that will help designers make empirically supported decisions about how to use spatiotemporal modulation to create (or avoid) the sensation of movement in ultrasound haptic designs.

\section{BACKGROUND}

Ultrasound haptic devices create mid-air haptic feedback through focused ultrasound, without the need to physically touch a device. This novel feedback modality was pioneered over a decade ago [22] and has advanced significantly since. For a more thorough discussion, we recommend a recent survey by Rakkolainen et al. [32].

A recent advance in this area was the adoption of spatiotemporal modulation, a rendering method where focal points are rapidly and continuously moved, enhancing perception over the presentation of stationary focal points $[12,37]$. This allowed more sophisticated 
tactile sensations, enhancing use of this feedback modality. A small body of research has investigated the perception of patterns rendered this way, which our contribution expands.

Takahashi et al. [35-37] investigated the perception of haptic patterns rendered using rapidly moving focal points (a method they called lateral modulation). Their results show that this leads to stronger feedback than simple amplitude modulation. Frier et al. [12] also found that moving focal points create stronger feedback. They investigated the perception of circles created by a rapidly moving focal point, finding a relationship between circle size, movement speed, and perceived intensity. Rutten et al. [33] investigated the just-noticeable difference (JND) for output intensity and focal point velocity when rendering haptic patterns. They found JNDs of $12.1 \%$ and $0.51 \mathrm{rev} \mathrm{s}^{-1}$, respectively. Ablart et al. [1] investigated perception of intensity, roughness, regularity and roundness, finding that the extent of each sensation varied with render frequency.

Circles are ideal for spatial modulation due to their uniform shape and lack of edge crossings or 'corners'. Other polygons like triangles and squares are more difficult to perceive. Hajas et al. [17] developed a more nuanced rendering method where a moving focal point will pause before changing direction. This was intended to emphasise the edges that make up a polygon and their orientation relative to each other, which was found to improve shape perception.

These works give insight into how patterns rendered by moving focal points are perceived. In this paper we contribute experiments that investigate the sensations of 'motion' that arise from this. Movement speed (or render frequency) is a key design parameter in a spatiotemporal modulated pattern, so a better understanding of this can inform the design of contactless haptic feedback.

\section{EXPERIMENT 1: SENSATION OF MOTION}

In this experiment, we take a first look at the perception of focal point motion. This is of interest because motion speed is a core parameter of spatiotemporal patterns. Studies by Frier et al. [12] and Takahashi et al. [36] have included 'slow' focal point velocities which may evoke a sense of motion, but not with the level of granularity necessary to understand how this is perceived. We address this gap in perceptual understanding by addressing the following research question: what is the relationship between rendering speed and the perceived sensation of motion? We investigate if users can perceive the sensation of motion and identify the range of speeds where motion is consistently perceived.

We investigated the movement of a single focal point along a circular trajectory, because circles are continuous paths and have no overlapping points. They have also been used in several ultrasound haptic interfaces for many purposes; e.g., spherical objects [21, 25, $29,30]$ or gesture feedback $[10,15,34]$. Our results can inform similar designs with empirical recommendations for creating (or avoiding) the sense of motion in spatiotemporal modulated patterns.

\subsection{Design}

We used an adaptive yes/no staircase procedure to vary focal point motion speed and estimate an upper bound for threshold motion speed: i.e., the maximum speed where motion can be perceived These procedures are used to estimate perceptual threshold values by 'narrowing in' on the threshold, using participant responses (yes/no) to adapt the presented stimuli. After a certain number of reversals on either side of the threshold, the staircase is terminated and a threshold can be estimated. The estimation is typically taken as the average of the most recent $n$ reversals. See work by García-Pérez [14] and Leek [28] for more information on adaptive psychometric procedures. We estimate the upper bound only; a pilot study found people stopped perceiving sensations entirely as the speed reduced, making a lower bound difficult to estimate.

We used a within-subjects design with four circle diameters: 4 , $5,6,7 \mathrm{~cm}$. We varied diameter to see if pattern size had an effect on perceived motion. Participants completed two blocks of three trials for each circle size. The order of the eight blocks was balanced using a Latin square design and there was a 15 second break between each task. After each block, we asked participants to describe the stimuli they just experienced. We presented trials in this manner to reduce habituation to stimuli. Participants wore headphones playing white noise to mask any audible hardware sounds.

For each trial, we continuously presented a circular pattern to the dominant hand, centred on the palm. Hands were held in place by a wrist rest, $15 \mathrm{~cm}$ above an Ultraleap UHEV1 device. The wrist rest was used to reduce fatigue and ensure the hand was in the correct position and orientation for the stimuli. We used the Ultraleap research SDK to implement this experiment. We used the maximum $16 \mathrm{kHz}$ sample rate of the device; varying the hardware refresh rate was out of the scope of this study, but should be investigated in future work to see if this affects perception of motion (e.g., [13]).

The movement speed of the focal point was varied using the staircase protocol, based on participant responses during the trial. The starting movement speed was $150 \mathrm{~cm} \mathrm{~s}^{-1}$. During each trial, we asked if participants felt a sensation of movement on their palm. If the answer was yes, we increased the speed by $18.24 \mathrm{~cm} \mathrm{~s}^{-1}$. If the answer was no, we decreased the speed by $20.94 \mathrm{~cm} \mathrm{~s}^{-1}$. The step sizes conformed to the recommended 0.871 ratio [14]. The starting value and step sizes were chosen after pilot tests.

Our procedure was based on a protocol by García-Pérez [14]. We used a 1-down/1-up staircase with six reversals; after six reversals, the staircase was terminated. The threshold was estimated using the movement speeds recorded at staircase reversals; the first two reversals were discarded, and the threshold estimate was the mean of the final four. For this version of the 1-down/1-up protocol, the estimated threshold is the $52.4 \%$ point on the psychometric function [14] (i.e., where perception was better than chance).

We recruited 14 participants (8 male, 6 female) who had never experienced ultrasound haptic feedback before. Their mean age was 28.8 years (sd 5.5). All were right handed. We used novice participants so that prior experience did not influence expectations. Participants were not told what the haptic stimuli were (i.e., circles).

\subsection{Results}

Our experiment data (see [11]) was used to calculate bootstrapped 95\% CIs for the threshold values for each circle diameter, using the $\mathrm{R}$ boot package [4]. Figure 2 shows the mean threshold values with 95\% CIs, measured in revolutions per second (i.e., how often the circle trajectory is traversed each second). While our experiment modelled movement speeds in $\mathrm{cm} \mathrm{s}^{-1}$, we report results in terms of 


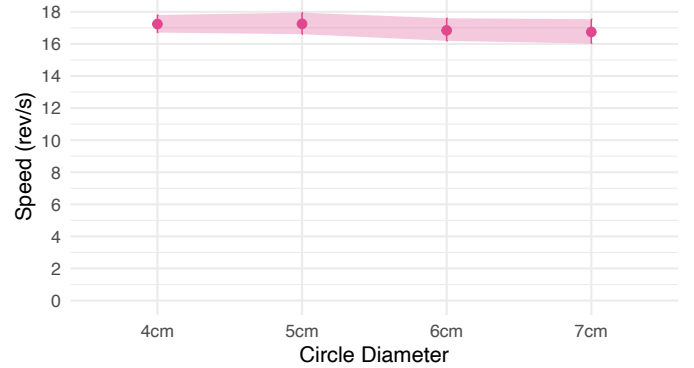

Figure 2: Mean threshold frequency with $95 \%$ CIs.

render frequency (in revolutions per second: rev s$^{-1}$ ) as we found this to be consistent across circle size.

The threshold render frequency was $17 \mathrm{rev} \mathrm{s}^{-1}$ (95\% CIs: [16.4, 17.7] $\mathrm{rev} \mathrm{s}^{-1}$ ), as shown in Figure 2. This is an estimate of the $52.4 \%$ point on the psychometric function [14]. At frequencies below this, users were more likely than chance to perceive movement on their palm, arising from the changing focal point position.

\subsection{Discussion}

We found that focal point frequencies up to $17 \mathrm{rev} \mathrm{s}^{-1}[16.4,17.7]$ can produce a perceptible sensation of movement on the palm, for circular patterns. The perceptual mechanisms responsible for spatiotemporal modulated patterns are not well understood yet, but this threshold provides insight into how these may be perceived.

Meissner corpuscles are rapidly-adapting touch receptors found on the palm. They are sensitive to low frequency vibrations from $1-50 \mathrm{~Hz}$ [24] and lateral motion [24]. Focal point movement below $17 \mathrm{rev} \mathrm{s}^{-1}$ threshold will likely stimulate Meissner corpuscles, since each point in the trajectory is stimulated up to 17 times per second. Pacinian corpuscles are another rapidly-adapting receptor, most sensitive to vibrations from $30-500 \mathrm{~Hz}$ [24]. These will also be stimulated by focal point motion, but are less sensitive than Meissner corpuscles at stimulation frequencies below $30 \mathrm{~Hz}[3,24]$.

A plausible explanation for the observed threshold was that it was a motion speed where Meissner corpuscles can perceive motion across the skin but Pacinian corpuscles are not strongly activated. Recent research suggests that separate somatosensory signals from Meissner and Pacinian corpuscles are integrated by the central nervous system, contributing to a combined percept of a single tactile event [27]. As the focal point motion speed increases beyond the threshold, the integrated signal becomes biased towards the stronger Pacinian corpuscle signal, leading to a reduced sensation of motion and increased sensation of vibration. Another way of looking at the threshold is as the point where the motion-sensitive receptors start to get overwhelmed by vibration-sensitive receptors.

The words used by participants to describe the haptic sensations were similar to "flutter", the term used in the literature to characterise the motion-like sensations perceived by Meissner corpuscles [2, 6]. Similar language was also reported in Obrist's study, where they used amplitude modulation to stimulate touch receptors with a static focal point at $16 \mathrm{~Hz}$ [31]. The sensations experienced by participants here likely corresponds to the flutter sensation.
Our results should generalise to other shapes rendered using spatiotemporal modulation. Complex polygons with changes in direction (e.g., at the corners of a rectangle) may stimulate certain areas of the palm for longer than others, leading to inconsistent stimulation along the trajectory. However, this variance will be subtle for most shapes, falling within the confidence intervals of our estimated threshold. More nuanced rendering methods for complex polygons (e.g., dynamic tactile pointers [17]) will require more investigation, to see how deliberately paused focal point movement impacts sensations of movement.

\section{EXPERIMENT 2: PERCEIVED MAGNITUDE}

In Experiment 1 we identified the threshold frequency for perceiving the sensation of motion in a dynamic circle pattern. Prior work suggests the perceived intensity also depends on frequency [1, 12]. The aim of our second experiment was therefore to investigate the relationship between perceived intensity and rendering frequency, below the $17 \mathrm{rev} \mathrm{s}^{-1}$ threshold. We address the following research question: what is the relationship between rendering speed and perceived intensity below this threshold? Answering this provides more insight into how these patterns are perceived and will help designers make an informed choice about how to convey a sensation that balances quality of motion with perceived intensity.

A study by Frier et al. [12] found that circles were strongest when rendered at 33-160 $\mathrm{rev} \mathrm{s}^{-1}$. Their study focused on higher frequencies, with only one data point below our threshold (13.3 $\mathrm{rev} \mathrm{s}^{-1}$ for a $4.77 \mathrm{~cm}$ circle). Our second experiment focuses on a much lower frequency range, with more granularity up to the motion threshold. Together, our results will give a better overall picture of the relationship between focal point movement (specifically rendering frequency) and perceived intensity.

\subsection{Design}

We used a magnitude estimation procedure, where participants make numerical judgements of perceived magnitude of stimuli. These procedures are used to understand the relative intensity of stimuli; in this case, the intensity of spatiotemporal modulated circles. See work by Jones and Tan for more information about magnitude estimation and its applicability to haptics research [23].

We used a within-subjects design with two circle sizes: 4 and $6 \mathrm{~cm}$ diameter. We chose this subset of circles from Experiment 1 to see if the perceived magnitude of the feedback varied with circle size. The experiment stimuli consisted of circles rendered at $\{5,7,9$, $11,13,15,17,19 \mathrm{rev} \mathrm{s}^{-1}$. These eight levels were chosen because they give good coverage of the perceptible frequency range up to the $17 \mathrm{rev} \mathrm{s}^{-1}$ threshold identified in Experiment 1, with one value above the threshold. The slowest was $5 \mathrm{rev} \mathrm{s}^{-1}$, because our pilot tests found this was the slowest frequency at which participants could reliably feel stimuli. Each stimuli was presented three times, giving 48 total ( 2 circle diameters, 8 frequencies, 3 repetitions). These were presented in a random order, with a two minute break enforced after every eight trials.

For each trial, we continuously presented one stimulus for five seconds to the palm of the participant's dominant hand. The circle was centred on the palm. Hands were placed on a wrist rest $15 \mathrm{~cm}$ 


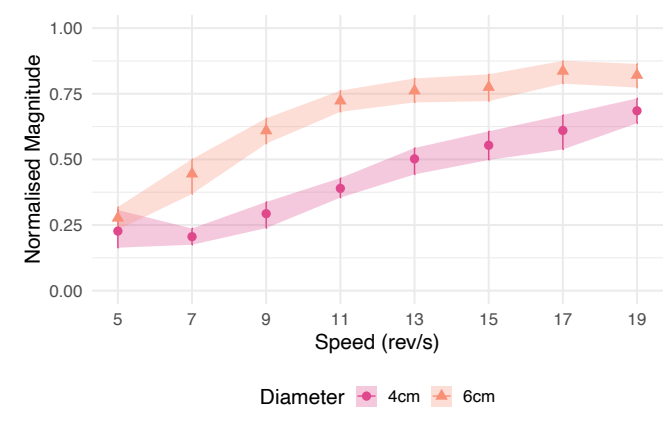

Figure 3: Mean magnitude estimates with 95\% CIs.

above an Ultraleap UHEV1 device. After the stimulus stopped, participants were asked if they felt anything. If they answered no, the next trial started. If they answered yes, they were asked to make a numerical judgement of the magnitude of the stimuli. They were instructed to use a numerical scale of their own choosing and were not given any reference stimuli. This is common to avoid biasing responses [23]. The next trial started when the participant was ready, no sooner than ten seconds after the previous.

We recruited 14 participants ( 7 male, 7 female, mean age 28 years, sd 6.9 years) who had never experienced ultrasound haptics before and did not take part in Experiment 1. All were right handed. We recruited people with no prior exposure to this technology so that prior experience did not bias expectations or provide a point for comparison. Participants were not told what the stimuli were.

\subsection{Results}

Participants chose their own rating scales to estimate magnitude. We normalised the estimates from each participant by dividing them by the mean of that individual's responses [23], mapping to a range of 0 to 1 for every participant. If a participant reported that they did not feel anything for a given stimulus, we assigned a rating of 0 . We calculated the geometric mean, rather than arithmetic mean, for the estimates, as recommended [23]. We used the same procedure as Experiment 1 to generate $95 \%$ CIs for the data (see [11]).

Magnitude estimates and 95\% CIs are shown in Figure 3. The mean intensity ratings for $4 \mathrm{~cm}$ and $6 \mathrm{~cm}$ circles were 0.42 and 0.66 , respectively. Estimates were significantly higher for the $6 \mathrm{~cm}$ circle: $\mathrm{t}(195)=20.5, \mathrm{p}<.001$. There were 10 undetected stimuli, reported by six participants; all of these were for the $4 \mathrm{~cm}$ circle at $5 \mathrm{rev} \mathrm{s}^{-1}$. One participant did not perceive any of the trials for that condition.

\subsection{Discussion}

Perceived magnitude increased as frequency increased, for both patterns. This is consistent with Frier's results [12], where intensity estimates increased towards a peak at 43-130 $\mathrm{rev} \mathrm{s}^{-1}$ (depending on diameter). If sensation of motion on the palm is desired, we suggest rendering circles with as high a frequency as possible, up to 17 $\mathrm{rev} \mathrm{s}^{-1}$. If sensation of motion is not desired (i.e., if a contiguous pattern is desired), we suggest using Frier's results [12] to identify a faster frequency that maximises intensity at the required size.

Our results show that perceived magnitude was greater for the larger circle size. Frier et al. [12] reported a similar finding for smaller circles $(1.59-4.77 \mathrm{~cm})$ at higher frequencies. They hypothesised that spatial summation was responsible for this finding; meaning larger circles stimulated a greater area of the hand, leading to a more intense tactile sensation. This could explain why the magnitude estimates were higher for our $6 \mathrm{~cm}$ circle.

Based on our findings, we expect circles smaller than $4 \mathrm{~cm}$ to feel weaker (i.e., lower magnitude) than our $4 \mathrm{~cm}$ pattern. We likewise expect magnitude to increase for circles larger than $6 \mathrm{~cm}$ (assuming they fit on the palm). In this experiment, stimuli were centred on the palm. We expect a subtle difference in intensity if circles were partially, or entirely, presented to the fingers, due to varying distribution and sensitivity of touch receptors on the hand.

Almost all stimuli were perceived by the participants, but some had difficulty detecting the $4 \mathrm{~cm}$ circle at the lowest frequency. We recommend a frequency where the normalised magnitude is at least 0.5 in Figure 3; for example, at least $9 \mathrm{rev} \mathrm{s}^{-1}$ for $6 \mathrm{~cm}$ or 13 $\mathrm{rev} \mathrm{s}^{-1}$ for $4 \mathrm{~cm}$. This means reliable detection is more likely and will ensure a good quality of haptic feedback. If differently sized shapes are being presented then perceptually-similar frequencies can be chosen; for example, $4 \mathrm{~cm}$ at $13 \mathrm{rev} \mathrm{s}^{-1}$ and $6 \mathrm{~cm}^{2} 7 \mathrm{rev} \mathrm{s}^{-1}$ overlap, so are likely to be perceived as having similar intensity.

\section{CONCLUSION}

We took a first look at the sensation of motion arising from focal point movement, as used in spatiotemporal [12] and lateral [37] rendering for ultrasound haptic devices. Focal point movement speed is an important parameter of these patterns and our results characterise its relationship with the sensation of movement and perceived intensity. For circular patterns, motion is perceived as a flutter-like sensation up to render frequencies of $17 \mathrm{rev} \mathrm{s}^{-1}$. Above this, people perceive a more constant haptic sensation.

Haptic circles are widely used in ultrasound haptic interfaces: e.g., for spherical virtual objects $[21,26,29,30]$ or to give feedback about mid-air gestures [7, 10, 15, 16, 34]. Our results can inform the design of ultrasound haptic interfaces, so that designers can create or avoid the sensation of tactile motion. Motion may be desirable for dynamic feedback: e.g., using below the $17 \mathrm{rev} \mathrm{s}^{-1}$ threshold to create moving patterns to indicate changing values or to accompany animated visual icons. Conversely, designers may wish to emphasise the contiguous outline of a virtual shape by rendering significantly above $17 \mathrm{rev} \mathrm{s}^{-1}$. Since perceived intensity scales with circle size and rendering frequency, our results can also be used to create perceptually similar haptic objects: i.e., by balancing size and frequency to yield similar intensity.

Our results also provide a foundation for future work into the perception and unique design opportunities arising from perceived motion: e.g., ability to identify movement direction (clockwise vs anticlockwise). Like others in this area [20,32], we encourage more work into the perception of ultrasound haptic feedback. A large and growing body of work is focused on the many novel applications of this feedback modality, but more work is needed to give designers and researchers empirical knowledge to inform their designs.

\section{ACKNOWLEDGMENTS}

This research was supported by the European Union Horizon 2020 research and innovation programme (FET Open \#737087: Levitate). 


\section{REFERENCES}

[1] Damien Ablart, William Frier, Hannah Limerick, Orestis Georgiou, and Marianna Obrist. 2019. Using Ultrasonic Mid-air Haptic Patterns in Multi-Modal User Experiences. In 2019 IEEE International Symposium on Haptic, Audio and Visual Environments and Games (HAVE). 1-6. https://doi.org/10.1109/HAVE.2019.8920969

[2] Victoria E. Abraira and David D. Ginty. 2013. The Sensory Neurons of Touch. Neuron 79, 4 (2013), 1-44. https://doi.org/10.1016/j.neuron.2013.07.051

[3] S. J. Bolanowski, George A. Gescheider, Ronald T. Verrillo, and C. M. Checkosky. 1988. Four channels mediate the mechanical aspects of touch. The fournal of the Acoustical Society of America 84, 5 (1988), 1680-1694. https://doi.org/10.1121/1. 397184

[4] Angelo Canty and Brian D. Ripley. 2019. R Package 'Boot'. https://cran.r project.org/web/packages/boot/

[5] Thomas Carter, Sue Ann Seah, Benjamin Long, Bruce Drinkwater, and Sriram Subramanian. 2013. UltraHaptics: Multi-Point Mid-Air Haptic Feedback for Touch Surfaces. In Proceedings of the 26th Symposium on User Interface Software and Technology - UIST '13. ACM Press, 505-514. https://doi.org/10.1145/2501988. 2502018

[6] Seungmoon Choi and Katherine J. Kuchenbecker. 2013. Vibrotactile display: Perception, technology, and applications. Proc. IEEE 101, 9 (2013), 2093-2104. https://doi.org/10.1109/JPROC.2012.2221071

[7] Brygida Dzidek, William Frier, Adam Harwood, and Richard Hayden. 2018. Design and Evaluation of Mid-Air Haptic Interactions in an Augmented Reality Environment. In Proceedings of EuroHaptics 2018 in LNCS 10894 - EuroHaptics '18. Springer International Publishing, 489-499. https://doi.org/10.1007/978-3-31993399-3_42

[8] Euan Freeman. 2021. Enhancing Ultrasound Haptics with Parametric Audio Effects. In Proceedings of the 23rd ACM International Conference on Multimodal Interaction - ICMI '21. ACM. https://doi.org/10.1145/3462244.3479951

[9] Euan Freeman, Ross Anderson, Julie Williamson, Graham Wilson, and Stephen Brewster. 2017. Textured Surfaces for Ultrasound Haptic Displays. In Proceedings of the 19th ACM International Conference on Multimodal Interaction - ICMI '17 Demos. ACM Press. https://doi.org/10.1145/3136755.3143020

[10] Euan Freeman, Dong-Bach Vo, and Stephen Brewster. 2019. HaptiGlow: Helping Users Position their Hands for Better Mid-Air Gestures and Ultrasound Haptic Feedback. In Proceedings of the IEEE World Haptics Conference 2019, the 8th foint Eurohaptics Conference and the IEEE Haptics Symposium. IEEE, 289-294. https: //doi.org/10.1109/WHC.2019.8816092

[11] Euan Freeman and Graham Wilson. 2021. User Study Data for "Perception of Ultrasound Haptic Focal Point Motion". https://doi.org/10.5281/zenodo.5142587

[12] William Frier, Damien Ablart, Jamie Chilles, Benjamin Long, Marcello Giordano, Marianna Obrist, and Sriram Subramanian. 2018. Using Spatiotemporal Modulation to Draw Tactile Patterns in Mid-air. In Proceedings of EuroHaptics 2018 Springer.

[13] William Frier, Dario Pittera, Damien Ablart, Marianna Obrist, and Sriram Subramanian. 2019. Sampling Strategy for Ultrasonic Mid-Air Haptics. In Proceedings of the 2019 CHI Conference on Human Factors in Computing Systems Proceedings CHI '19. ACM Press, Paper 121. https://doi.org/10.1145/3290605.3300351

[14] Miguel A. García-Pérez. 2001. Yes-No Staircases with Fixed Step Sizes: Psychometric Properties and Optimal Setup. Optometry and Vision Science 78, 1 (2001), 56-64. https://doi.org/10.1097/00006324-200101010-00015

[15] Orestis Georgiou and Daniel Griffiths. 2017. Haptic In-vehicle Gesture Controls. In Adjunct Proceedings of the 9th International ACM Conference on Automotive User Interfaces and Interactive Vehicular Applications - AutomotiveUI '17. ACM Press, 233-238. https://doi.org/10.1145/3131726.3132045

[16] Orestis Georgiou, Craig Jeffrey, Ziyuan Chen, Bao Xiao Tong, Shing Hei Chan, Boyin Yang, Adam Harwood, and Tom Carter. 2018. Touchless Haptic Feedback for VR Rhythm Games. In Proceedings of 25th IEEE Conference on Virtual Reality and $3 D$ User Interfaces - IEEE VR '18. IEEE, 2-3. https://doi.org/10.1109/VR.2018. 8446619

[17] Daniel Hajas, Dario Pittera, Antony Nasce, Orestis Georgiou, and Marianna Obrist. 2020. Mid-Air Haptic Rendering of 2D Geometric Shapes with a Dynamic Tactile Pointer. IEEE Transactions on Haptics 13, 1 (2020), 1-12. https://doi.org/ 10.1109/TOH.2020.2966445

[18] Ryuji Hirayama, Diego Martinez Plasencia, Nobuyuki Masuda, and Sriram Subramanian. 2019. A volumetric display for visual, tactile and audio presentation using acoustic trapping. Nature 575 (2019). https://doi.org/10.1038/s41586-019-1739-5

[19] Takayuki Hoshi. 2011. Development of aerial-input and aerial-tactile-feedback system. In Proceedings of the 2011 IEEE World Haptics Conference. IEEE, 569-573. https://doi.org/10.1109/WHC.2011.5945548

[20] Thomas Howard, Gerard Gallagher, Anatole Lécuyer, Claudio Pacchierotti, and Maud Marchal. 2019. Investigating the Recognition of Local Shapes Using Mid-air Ultrasound Haptics. Proceedings of the 2019 IEEE World Haptics Conference - WHC '19 (2019), 503-508. https://doi.org/10.1109/WHC.2019.8816127

[21] Seki Inoue and Hiroyuki Shinoda. 2014. A pinchable aerial virtual sphere by acoustic ultrasound stationary wave. In IEEE Haptics Symposium - HAPTICS '14. IEEE, 89-92. https://doi.org/10.1109/HAPTICS.2014.6775437
[22] Takayuki Iwamoto, Mari Tatezono, and Hiroyuki Shinoda. 2008. Non-contact method for producing tactile sensation using airborne ultrasound. In Proceedings of EuroHaptics 2008. Springer, 504-513. https://doi.org/10.1007/978-3-540-69057364

[23] Lynette A. Jones and Hong Z. Tan. 2013. Application of Psychophysical Techniques to Haptic Research. IEEE Transactions on Haptics 6, 3 (2013), 268-284. https://doi.org/10.1109/TOH.2012.74

[24] Eric R. Kandel, James H. Schwartz, Thomas M. Jessell, Steven A. Siegelbaum, and A. J. Hudspeth. 2012. Touch. In Principles of Neural Science (5th ed.). McGraw-Hill Publishing, Chapter 23, 498-529.

[25] Georgios Korres, Tamas Aujeszky, and Mohamad Eid. 2017. Characterizing Tactile Rendering Parameters For Ultrasound Based Stimulation. In Proceedings of IEEE World Haptics Conference - WHC '17. IEEE, 293-298. https://doi.org/10.1109/ WHC.2017.7989917

[26] Georgios Korres and Mohamad Eid. 2016. Haptogram: Ultrasonic Point-Cloud Tactile Stimulation. IEEE Access 4 (2016), 7758 - 7769. https://doi.org/10.1109/ ACCESS.2016.2608835

[27] Scinob Kuroki, Junji Watanabe, and Shin'ya Nishida. 2017. Integration of vibrotactile frequency information beyond the mechanoreceptor channel and somatotopy. Scientific Reports 7, 1 (2017), 1-13. https://doi.org/10.1038/s41598-017-02922-7

[28] Marjorie R. Leek. 2001. Adaptive procedures in psychophysical research. Perception \& psychophysics 63, 8 (2001), 1279-1292. https://doi.org/10.3758/BF03194543

[29] Benjamin Long, Sue Ann Seah, Tom Carter, and Sriram Subramanian. 2014. Rendering Volumetric Haptic Shapes in Mid-Air using Ultrasound. ACM Transactions on Graphics 33, 6 (2014), Article 181. https://doi.org/10.1145/2661229.2661257

[30] Yasutoshi Makino, Yoshikazu Furuyama, Seki Inoue, and Hiroyuki Shinoda. 2016. HaptoClone (Haptic-Optical Clone) for Mutual Tele-Environment by Real-time 3D Image Transfer with Midair Force Feedback. In Proceedings of the SIGCHI Conference on Human Factors in Computing Systems - CHI '16. ACM Press, 19801990. https://doi.org/10.1145/2858036.2858481

[31] Marianna Obrist, Sue Ann Seah, and Sriram Subramanian. 2013. Talking about Tactile Experiences. In Proceedings of the SIGCHI Conference on Human Factors in Computing Systems - CHI '13. ACM Press, 1659-1668. https://doi.org/10.1145/ 2470654.2466220

[32] Ismo Rakkolainen, Euan Freeman, Antti Sand, Roope Raisamo, and Stephen Brewster. 2021. A Survey of Mid-Air Ultrasound Haptics and Its Applications. IEEE Transactions on Haptics 14, 1 (2021), 2-19. https://doi.org/10.1109/TOH. 2020.3018754

[33] Isa Rutten, William Frier, and David Geerts. 2020. Discriminating Between Intensities and Velocities of Mid-Air Haptic Patterns. In Proceedings of the International Conference on Human Haptic Sensing and Touch Enabled Computer Applications - EuroHaptics 2020. Springer International Publishing, 78-86. https://doi.org/10.1007/978-3-030-58147-3 9

[34] Gözel Shakeri, John H Williamson, and Stephen Brewster. 2018. May the Force Be with You: Ultrasound Haptic Feedback for Mid-Air Gesture Interaction in Cars. In Proceedings of Automotive UI 2018 - AutoUI '18. ACM Press.

[35] Ryoko Takahashi, Keisuke Hasegawa, and Hiroyuki Shinoda. 2018. Lateral Modulation of Midair Ultrasound Focus for Intensified Vibrotactile Stimuli. In Proceedings of EuroHaptics 2018 in LNCS 10894 - EuroHaptics '18. Springer International Publishing, 276-288. https://doi.org/10.1007/978-3-319-93399-3_25

[36] Ryoko Takahashi, Keisuke Hasegawa, and Hiroyuki Shinoda. 2019. Tactile Stimulation by Repetitive Lateral Movement of Midair Ultrasound Focus. IEEE Transactions on Haptics (2019). https://doi.org/10.1109/TOH.2019.2946136

[37] Ryoko Takahashi, Keisuke Hasegawa, and Hiroyuki Shinoda. 2020. Tactile Stimulation by Repetitive Lateral Movement of Midair Ultrasound Focus. IEEE Transactions on Haptics 13, 2 (2020), 334-342. https://doi.org/10.1109/TOH.2019.2946136 